\title{
ERRATA
}

\section{Erratum: Autosolitons in InSb in a magnetic field [Semiconductors 32, 625-628 (June 1988)]}

I. K. Kamilov, A. A. Stepurenko, and A. S. Kovalev

Kh. I. Amirkhanov Physics Institute, 367003 Makhachkala, Russia

Fiz. Tekh. Poluprovodn. 32, 1535 (December 1998)

[S1063-7826(98)01512-9]

The sentence beginning on the 14th line from the top of the right-hand column on p. 627 reads: It turned out that the velocity of the autosoliton decreases as it moves into the hotter region of the EHP. It should read: It turned out that the velocity of the autosoliton increases as it moves into the hotter region of the EHP and that the velocity of the autosoliton decreases as it moves into the colder region of the EHP. 